\title{
Distributed Two-Stage Multi-Cell Precoding
}

\author{
Ayswarya Padmanabhan, Antti Tölli, and Italo Atzeni \\ Centre for Wireless Communications (CWC), University of Oulu, Finland \\ Emails: \{ayswarya.padmanabhan, antti.tolli, italo.atzeni\}@oulu.fi
}

\begin{abstract}
This paper proposes a distributed downlink precoding design for multi-cell massive multiple-input multiple-output systems. Two-stage precoding is adopted assuming that the user equipments (UEs) in each base station (BS) are grouped according to matching channel statistics. In this regard, the channel dimension is first reduced by means of statistical, group-specific processing. Subsequently, the UE-specific inner beamformers (IBFs) are optimized based on the resulting (lower-dimensional) effective channels, with sensibly reduced computational complexity. We begin by formulating a centralized IBF design that derives from iteratively solving the Karush-Kuhn-Tucker conditions of the weighted sum rate maximization problem. Then, we propose a distributed algorithm where inter-cell interference (ICI) terms and dual variables are periodically exchanged among neighboring BSs via backhaul signaling, whereas the inter-group interference (IGI) within each BS is handled locally. Furthermore, the ICI updates between the BSs are allowed to take place less frequently than the local IGI updates. Numerical results show that enabling backhaul signaling every 5-10 iterations of the algorithm yields a remarkably small performance loss with respect to the case with full information exchange between the BSs.
\end{abstract}

\section{INTRODUCTION}

Massive multiple-input multiple-output (MIMO) is among the key enabling technologies of the upcoming fifth-generation (5G) cellular standard [1]. A critical aspect of massive MIMO communications is that the high dimensionality of the resulting channels sensibly complicates the channel state information (CSI) acquisition and the matrix operations involved in the computation of the transmission strategies. For this reason, approaches seeking complexity reduction have gained special attention in recent years, ranging from fully-digital [2] to hybrid (analog/digital) precoding techniques [3].

In fully-digital two-stage precoding, a statistical outer beamformer (OBF) provides pre-beams for different user equipments (UEs) or propagation paths, effectively reducing the channel dimension while accounting only for the strongest directions. This allows to decrease the number of significant channel coefficients per UE, resulting in a more accurate effective channel estimation. After designing the OBF, an inner beamformer (IBF) is subsequently applied for spatial multiplexing onto the effective beam-domain channel of lower dimension. Note that the OBFs requires less frequent updates as compared to the IBFs due to the longer-term validity of statistical CSI.

The most noteworthy example of fully-digital two-stage precoding is joint spatial division and multiplexing (JSDM) [2],

This work was supported by the Academy of Finland under grant no. 312648 and 318927 (6Genesis Flagship). which builds on grouping UEs with similar covariance properties to design the OBFs. More precisely, group-specific processing is implemented by selecting the discrete Fourier transform (DFT) beams corresponding to the angular positions of the UE groups, which provides a sum-rate performance comparable to that obtained with full CSI. In [4]-[6], JSDM is studied extensively for UE grouping, whereas in [7] OBFs and IBFs are used to tackle the intra- and inter-cell interference (ICI), respectively. Two-stage precoding is considered in [8] with various heuristic OBF strategies and the sum rate is analyzed as a function of number of statistical beams.

In this paper, we propose a two-stage downlink precoding design for multi-cell massive MIMO systems. Assuming that the UEs are collocated geographically and grouped on the basis of similar channel statistics, the channel dimension is first reduced by means of a statistical, group-specific OBF matrix obtained via greedy selection of DFT beams (as in [9], [10]). Then, the UE-specific IBF vectors are optimized based on the effective beam-domain channels of lower dimension according to the weighted sum rate maximization (WSRM) criterion with per-group transmit power constraints. To come up with a lowcomplexity method amenable for practical implementation, we present a centralized IBF design obtained by iteratively solving the Karush-Kuhn-Tucker (KKT) conditions of the WSRM problem, as an extension of the single-cell case in [10]. Finally, we propose a distributed algorithm where the ICI is controlled by periodically exchanging real-valued scalars among neighboring base stations (BSs) via backhaul signaling, whereas the inter-group interference (IGI) within each BS is handled locally. Furthermore, assuming stringent backhaul constraints, the ICI updates among the BSs are allowed to occur on a longer-term basis with respect to the local IGI updates. The proposed method provides insight into the tradeoff between dimensionality reduction and sum-rate performance in multicell systems with group-specific processing.

\section{SySTEM MOdEL}

Consider the downlink of a multi-cell massive MIMO system where a set of BSs $\mathcal{B}=\{1, \ldots, B\}$, each equipped with $N_{\mathrm{T}}$ transmit antennas, serves a set of single-antenna UEs $\mathcal{K}=\{1, \ldots, K\}$ as in Fig. 1 . As the UEs tend to be geographically collocated in clusters, we consider UE grouping for designing the transmission strategies at the BSs. Accordingly, let $\mathcal{G}=\{1, \ldots, G\}$ denote the set of UE groups and let $\mathcal{G}_{b} \subset \mathcal{G}$ be the subset of groups associated with BS $b \in \mathcal{B}$. Furthermore, we define $\mathcal{K}_{g} \subset \mathcal{K}$ as the subset 


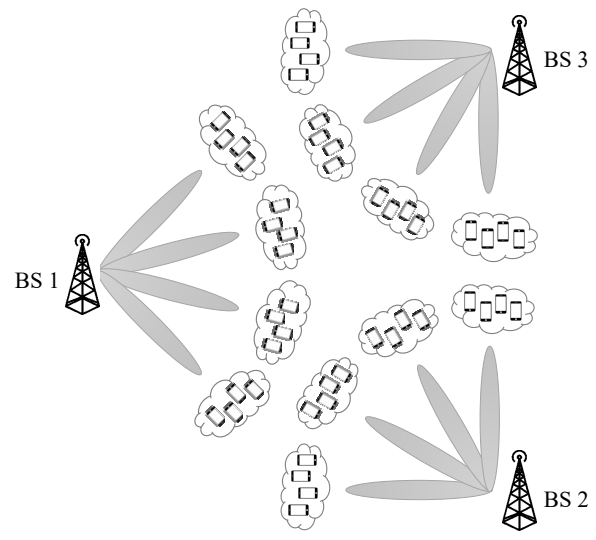

Fig. 1. Considered multi-cell system with UE grouping.

of UEs in group $g \in \mathcal{G}$ and $\mathcal{K}^{(b)}=\cup_{g \in \mathcal{G}_{b}} \mathcal{K}_{g}$ as the subset of UEs served by BS $b \in \mathcal{B}$. We use $b_{k}$ and $g_{k}$ to indicate, respectively, the serving $\mathrm{BS}$ and the group of $\mathrm{UE} k \in \mathcal{K}$.

The channel between BS $b \in \mathcal{B}$ and UE $k \in \mathcal{K}$ is denoted by $\mathbf{h}_{b, k} \in \mathbb{C}^{N_{\mathrm{T}} \times 1}$. To model the channels based on the UEs' location in the azimuthal direction, we adopt the one-ring model [11] and assume that each BS is equipped with a uniform linear array characterized by half-wavelength spacing between antennas. Hence, letting $\mathbf{a}(\theta)=\left[1, e^{-j \pi \cos (\theta)}, \ldots, e^{-j \pi \cos (\theta)\left(N_{\mathrm{T}}-1\right)}\right]^{\mathrm{T}}$ denote the steering vector for the angle of departure $\theta$, we have

$$
\mathbf{h}_{b, k}=\frac{d_{b, k}^{-\eta / 2}}{\sqrt{L}} \sum_{l=1}^{L} e^{j \phi_{b, k, l}} \mathbf{a}\left(\theta_{b, k, l}\right)
$$

where $d_{b, k}$ is the distance from BS $b \in \mathcal{B}$ to UE $k \in \mathcal{K}, \eta>2$ represents the pathloss exponent, $L$ is number of scatterers uniformly distributed around UE $k$ with angular spread $\sigma_{b, k}$, and $\phi_{b, k, l}$ is the random phase introduced by scatterer $l$.

As in our previous works [9], [10], the BSs adopt a twostage precoding design that achieves a remarkable complexity reduction as compared to traditional MIMO transmission techniques by exploiting the geographical distribution of the UEs in clusters. In this setting, the beamforming vector for each UE is characterized as the combination of a statistical, groupspecific OBF matrix and an instantaneous, UE-specific IBF vector. Let $S_{g}$ be the number of statistical beams corresponding to UE group $g \in \mathcal{G}$ and let $S^{(b)}=\sum_{g \in \mathcal{G}_{b}} S_{g}$ represent the total number of statistical beams used by BS $b \in \mathcal{B}$. The beamforming vector for UE $k \in \mathcal{K}$ is thus given by $\mathbf{v}_{k}=\mathbf{B}_{g_{k}} \mathbf{w}_{k} \in \mathbb{C}^{N_{\mathrm{T}} \times 1}$, where $\mathbf{B}_{g_{k}} \in \mathbb{C}^{N_{\mathrm{T}} \times S_{g_{k}}}$ is the OBF matrix containing the statistical beams corresponding to group $g_{k} \in \mathcal{G}_{b_{k}}$ and $\mathbf{w}_{k} \in \mathbb{C}^{S_{g_{k}} \times 1}$ is the IBF vector. For convenience, we use $\overline{\mathbf{h}}_{b, g, k}=\mathbf{B}_{g}^{\mathrm{H}} \mathbf{h}_{b, k} \in \mathbb{C}^{S_{g} \times 1}$ to denote the effective channel from BS $b \in \mathcal{B}$ to UE $k \in \mathcal{K}$ after precoding with the OBF matrix corresponding to group $g \in \mathcal{G}_{b}$.

For the OBF design, we use the greedy beam selection technique from [9], which is briefly described next. Let $\mathbf{H}^{(b)}=\left[\mathbf{h}_{b, \mathcal{K}^{(b)}(1)}, \ldots, \mathbf{h}_{b, \mathcal{K}^{(b)}\left(\left|\mathcal{K}^{(b)}\right|\right)}\right]$ be the matrix including the channels of all the UEs served by BS $b \in \mathcal{B}$ and let $\mathbf{R}^{(b)}=\mathbb{E}\left[\mathbf{H}^{(b)}\left(\mathbf{H}^{(b)}\right)^{\mathrm{H}}\right]$, which can be decomposed via eigenvalue decomposition as $\mathbf{R}^{(b)}=\mathbf{U}^{(b)} \boldsymbol{\Lambda}^{(b)}\left(\mathbf{U}^{(b)}\right)^{\mathrm{H}}$. Likewise, let $\mathbf{H}_{g}=\left[\mathbf{h}_{b, \mathcal{K}_{g}(1)}, \ldots, \mathbf{h}_{b, \mathcal{K}_{g}\left(\left|\mathcal{K}_{g}\right|\right)}\right]$ be the matrix comprising the channels of all the UEs in group $g \in \mathcal{G}_{b}$ and let $\mathbf{R}_{g}=$ $\mathbb{E}\left[\mathbf{H}_{g} \mathbf{H}_{g}^{\mathrm{H}}\right]$, which can be decomposed as $\mathbf{R}_{g}=\mathbf{U}_{g} \boldsymbol{\Lambda}_{g} \mathbf{U}_{g}^{\mathrm{H}}$. As the number of UEs served by BS $b \in \mathcal{B}$ increases, the probability of finding a UE in any azimuthal direction follows the uniform distribution in $[-\pi / 2, \pi / 2]$. Hence, in such limiting case, the columns of $\mathbf{U}^{(b)} \in \mathbb{C}^{N_{\mathrm{T}} \times S^{(b)}}$ can be approximated by the columns of the DFT matrix $\mathbf{D}=\left[\mathbf{d}_{1}, \ldots, \mathbf{d}_{N_{\mathrm{T}}}\right] \in \mathbb{C}^{N_{\mathrm{T}} \times N_{\mathrm{T}}}$, with $\mathbf{d}_{n}=\left[1, e^{-j 2 \pi n / N_{\mathrm{T}}}, \ldots, e^{-j 2 \pi n\left(N_{\mathrm{T}}-1\right) / N_{\mathrm{T}}}\right]^{\mathrm{T}} / \sqrt{N_{\mathrm{T}}}$. It follows that the columns of $\mathbf{U}_{g} \in \mathbb{C}^{N_{\mathrm{T}} \times S_{g}}$ can be approximated by a properly chosen subset of DFT vectors. To do so, we initialize $\mathcal{D}=\left\{1, \ldots, N_{\mathrm{T}}\right\}, \mathcal{D}_{g}=\emptyset$, and iteratively select the DFT vectors corresponding to the $S_{g}$ strongest signal paths for group $g \in \mathcal{G}_{b}$ as

$$
\begin{aligned}
m & =\underset{n}{\operatorname{argmax}}\left(\mathbf{d}_{n}^{\mathrm{H}} \mathbf{R}_{g} \mathbf{d}_{n}\right), \quad \forall n \in \mathcal{D}, \\
\mathcal{D}_{g} & =\mathcal{D}_{g} \cup\{m\}, \quad \mathcal{D}=\mathcal{D} \backslash\{m\} .
\end{aligned}
$$

Upon finding the subset $\mathcal{D}_{g}$, the group-specific OBF matrix is finally given by $\mathbf{B}_{g}=\left[\mathbf{d}_{\mathcal{D}_{g}(1)}, \ldots, \mathbf{d}_{\mathcal{D}_{g}\left(S_{g}\right)}\right]$.

The received signal at UE $k \in \mathcal{K}$ is given as in (3) at the bottom of the page, where $x_{k} \in \mathbb{C}$ is the transmitted symbol with $\mathbb{E}\left[x_{k} x_{k}^{\mathrm{H}}\right] \leq 1$ and $n_{k} \sim \mathcal{C N}\left(0, N_{0}\right), \forall k \in \mathcal{K}$, is the additive white Gaussian noise. The first term in the RHS of (3) is the desired signal, the second and third terms represent, respectively, the intra-group interference and the IGI from the serving BS, and the fourth term is the ICI caused by the neighboring BSs. The resulting signal-to-interference-plusnoise ratio (SINR) is given as in (4) at the bottom of the page.

To optimize the IBF vectors $\left\{\mathbf{w}_{k}\right\}_{k \in \mathcal{K}}$, we consider the WSRM problem with per-group transmit power constraint, i.e.,

$$
\begin{array}{ll}
\underset{\left\{\mathbf{w}_{k}\right\}_{k \in \mathcal{K}}}{\operatorname{maximize}} & \sum_{k \in \mathcal{K}} \alpha_{k} \log _{2}\left(1+\gamma_{k}\right) \\
\text { subject to } & \sum_{k \in \mathcal{K}_{g}}\left\|\mathbf{B}_{g} \mathbf{w}_{k}\right\|^{2} \leq \frac{P_{\mathrm{T}}}{G^{(b)}}, \quad \forall g \in \mathcal{G}_{b}, \forall b \in \mathcal{B}
\end{array}
$$

$$
\begin{gathered}
y_{k}=\overline{\mathbf{h}}_{b_{k}, g_{k}, k}^{\mathrm{H}} \mathbf{w}_{k} x_{k}+\sum_{\bar{k} \in \mathcal{K}_{g_{k}} \backslash\{k\}} \overline{\mathbf{h}}_{b_{k}, g_{k}, k}^{\mathrm{H}} \mathbf{w}_{\bar{k}} x_{\bar{k}}+\sum_{\bar{g} \in \mathcal{G}_{b} \backslash\left\{g_{k}\right\}} \sum_{\bar{k} \in \mathcal{K}_{\bar{g}}} \overline{\mathbf{h}}_{b_{k}, \bar{g}, k}^{\mathrm{H}} \mathbf{w}_{\bar{k}} x_{\bar{k}}+\sum_{\bar{b} \in \mathcal{B} \backslash\left\{b_{k}\right\}} \sum_{\bar{g} \in \mathcal{G}_{\bar{b}}} \sum_{\bar{k} \in \mathcal{K}_{\bar{g}}} \overline{\mathbf{h}}_{\bar{b}, \bar{g}, k}^{\mathrm{H}} \mathbf{w}_{\bar{k}} x_{\bar{k}}+n_{k}, \\
\sum_{k}=\frac{\left|\overline{\mathbf{h}}_{b_{k}, g_{k}, k}^{\mathrm{H}} \mathbf{w}_{k}\right|^{2}}{\left.\left|\sum_{\bar{k} \in \mathcal{K}_{g_{k}} \backslash\{k\}}\right| \overline{\mathbf{h}}_{b_{k}, g_{k}, k}^{\mathrm{H}} \mathbf{w}_{\bar{k}}\right|^{2}+\sum_{\bar{g} \in \mathcal{G}_{b} \backslash\left\{g_{k}\right\}} \sum_{\bar{k} \in \mathcal{K}_{\bar{g}}}\left|\overline{\mathbf{h}}_{b_{k}, \bar{g}, k}^{\mathrm{H}} \mathbf{w}_{\bar{k}}\right|^{2}+\sum_{\bar{b} \in \mathcal{B} \backslash\left\{b_{k}\right\}} \sum_{\bar{g} \in \mathcal{G}_{\bar{b}}} \sum_{\bar{k} \in \mathcal{K}_{\bar{g}}}\left|\overline{\mathbf{h}}_{\bar{g}, \bar{g}, k}^{\mathrm{H}} \mathbf{w}_{\bar{k}}\right|^{2}+N_{0}}
\end{gathered}
$$


where the weight $\alpha_{k} \geq 0$ determines the scheduling priority of UE $k \in \mathcal{K}, G^{(b)}=\left|\mathcal{G}_{b}\right|$ is the number of UE groups in $\mathrm{BS} b \in \mathcal{B}$, and $P_{\mathrm{T}}$ is the maximum transmit power at the BSs.

\section{ITERATIVE IBF DESIGN}

The IBF design for the multi-cell system described in Section II introduces major implementation and signaling challenges with respect to the single-cell scenario considered in our previous works [9], [10]. In this regard, to tackle the WSRM problem in (5), each BS is required to maximize the desired signal power at the served UEs while controlling the ICI caused at the UEs in the neighboring BSs. We begin by formulating a centralized IBF design in Section III-A, which derives from iteratively solving the KKT conditions of the WSRM problem. Then, in Section III-B, we propose a distributed IBF design where the ICI is handled via limited exchange of real-valued UE-specific scalars among the BSs.

\section{A. Centralized IBF Design}

In the centralized setting, the full CSI of the whole network is collected by a central unit, which then computes the IBF vectors without any further information exchange between the BSs. To solve the WSRM problem in (5), we begin by relaxing the interference-plus-noise term in the denominator of (4) as

$$
\begin{aligned}
\sum_{\bar{k} \in \mathcal{K}_{g_{k}} \backslash\{k\}}\left|\overline{\mathbf{h}}_{b_{k}, g_{k}, k}^{\mathrm{H}} \mathbf{w}_{\bar{k}}\right|^{2}+\sum_{\bar{g} \in \mathcal{G}_{b} \backslash\left\{g_{k}\right\}} \sum_{\bar{k} \in \mathcal{K}_{\bar{g}}}\left|\overline{\mathbf{h}}_{b_{k}, \bar{g}, k}^{\mathrm{H}} \mathbf{w}_{\bar{k}}\right|^{2} \\
+\sum_{\bar{b} \in \mathcal{B} \backslash\left\{b_{k}\right\}} \sum_{\bar{g} \in \mathcal{G}_{\bar{b}}} \sum_{\bar{k} \in \mathcal{K}_{\bar{g}}}\left|\overline{\mathbf{h}}_{\bar{b}, \bar{g}, k}^{\mathrm{H}} \mathbf{w}_{\bar{k}}\right|^{2}+N_{0} \leq \beta_{k}
\end{aligned}
$$

and rewrite (5) as (cf. [9])

$$
\begin{aligned}
\underset{\left\{\gamma_{k}, \beta_{k}, \mathbf{w}_{k}\right\}_{k \in \mathcal{K}}}{\operatorname{maximize}} \sum_{k \in \mathcal{K}} \alpha_{k} \log _{2}\left(1+\gamma_{k}\right) & \\
\text { subject to } & \\
p_{k}: \frac{\left|\overline{\mathbf{h}}_{b_{k}, g_{k}, k}^{\mathrm{H}} \mathbf{w}_{k}\right|^{2}}{\beta_{k}} \geq \gamma_{k}, & \forall k \in \mathcal{K} \\
q_{k}:(6), & \forall k \in \mathcal{K} \\
s_{g}: \sum_{k \in \mathcal{K}_{g}}\left\|\mathbf{B}_{g} \mathbf{w}_{k}\right\|^{2} \leq \frac{P_{\mathrm{T}}}{G^{(b)}}, & \forall g \in \mathcal{G}_{b}, \forall b \in \mathcal{B}
\end{aligned}
$$

where we have introduced the dual variables $p_{k}, q_{k}$, and $s_{g}$ associated with each constraint in (7a), (7b) and (7c), respectively. However, this reformulation is still nonconvex due to the constraints in (7a). To efficiently solve the problem in (7), we resort to successive convex approximation, whereby each nonconvex constraint is replaced by a sequence of approximate convex functions and the approximate problem is solved iteratively until convergence [12]. To do so, we replace the quadratic-over-linear term in the LHS of (7a) by its firstorder Taylor approximation around $\left\{\mathbf{w}_{k}^{(i)}, \beta_{k}^{(i)}\right\}$, given by [13]

$$
\begin{aligned}
& f_{k}^{(i)}\left(\mathbf{w}_{k}, \beta_{k} ; \mathbf{w}_{k}^{(i)}, \beta_{k}^{(i)}\right)=\frac{2\left(\mathbf{w}_{k}^{(i)}\right)^{\mathrm{H}} \overline{\mathbf{h}}_{b_{k}, g_{k}, k} \overline{\mathbf{h}}_{b_{k}, g_{k}, k}^{\mathrm{H}}}{\beta_{k}^{(i)}} \\
& \quad \times\left(\mathbf{w}_{k}-\mathbf{w}_{k}^{(i)}\right)+\frac{\left|\overline{\mathbf{h}}_{b_{k}, g_{k}, k}^{\mathrm{H}} \mathbf{w}_{k}^{(i)}\right|^{2}}{\beta_{k}^{(i)}}\left(1-\frac{\beta_{k}-\beta_{k}^{(i)}}{\beta_{k}^{(i)}}\right) .
\end{aligned}
$$

For the single-cell scenario, the performance of the iterative optimization using the KKT conditions with fixed IGI dual variables was observed in [9] to be remarkably close to that obtained with the centralized IBF design. Motivated by this, we tackle the WSRM problem in (5) by iteratively solving the system of KKT conditions of the problem in (7), whose $i$ th iteration for UE $k \in \mathcal{K}$ reads as in (9) at the bottom of the page. To initialize the algorithm, we fix $\mathbf{w}_{k}^{(0)}$ to some feasible IBF vector and obtain $\gamma_{k}^{(0)}$ and $\beta_{k}^{(0)}$, which are then used to derive $p_{k}^{(1)}$ and $q_{k}^{(1)}$. Note that $s_{g}^{(i)}$ can be computed by means of bisection search so as to satisfy the per-group transmit power constraint.

The computational complexity of the proposed iterative downlink precoding design mostly stems from computation of the IBF vectors, since the OBF matrices are updated on a longer-term basis (i.e., whenever the channel statistics change). From (9b), we observe that the computation of $\mathbf{w}_{k}$ consists mainly of matrix multiplications and inversions, whose complexity highly depends on the dimension of the involved matrices. Hence, due to the dimensionality reduction (from $N_{\mathrm{T}}$-dimensional channels to $S_{g}$-dimensional effective channels) brought by the group-specific processing, the com-

$$
\begin{aligned}
p_{k}^{(i)}= & \frac{\alpha_{k}}{1+\gamma_{k}^{(i-1)}}, \quad q_{k}^{(i)}=\frac{\left|\overline{\mathbf{h}}_{b_{k}, g_{k}, \mathbf{w}_{k}^{\mathrm{H}}}^{\mathrm{H}} \mathbf{w}_{k}^{(i-1)}\right|^{2}}{\left(\beta_{k}^{(i-1)}\right)^{2}} p_{k}^{(i)}, \\
\mathbf{w}_{k}^{(i)}= & \left(s_{g_{k}}^{(i)} \mathbf{B}_{g_{k}}^{\mathrm{H}} \mathbf{B}_{g_{k}}+\sum_{\bar{k} \in \mathcal{K}_{g_{k}} \backslash\{k\}} q_{\bar{k}}^{(i)} \overline{\mathbf{h}}_{b_{k}, g_{k}, \bar{k}} \overline{\mathbf{h}}_{b_{k}, g_{k}, \bar{k}}^{\mathrm{H}}+\sum_{\bar{g} \in \mathcal{G}_{b} \backslash\left\{g_{k}\right\} \bar{k} \in \mathcal{K}_{\bar{g}}} q_{\bar{k}}^{(i)} \overline{\mathbf{h}}_{b_{k}, g_{k}, \bar{k}} \overline{\mathbf{h}}_{b_{k}, g_{k}, \bar{k}}^{\mathrm{H}}\right. \\
& \left.+\sum_{\bar{b} \in \mathcal{B} \backslash\left\{b_{k}\right\}} \sum_{\bar{g} \in \mathcal{G}_{\bar{b}}} \sum_{\bar{k} \in \mathcal{K}_{\bar{g}}} q_{\bar{k}}^{(i)} \overline{\mathbf{h}}_{b_{k}, g_{k}, \bar{k}} \overline{\mathbf{h}}_{b_{k}, g_{k}, \bar{k}}^{\mathrm{H}}\right)^{-1} \frac{p_{k}^{(i)} \overline{\mathbf{h}}_{b_{k}, g_{k}, k} \overline{\mathbf{h}}_{b_{k}, g_{k}, k}^{\mathrm{H}} \mathbf{w}_{k}^{(i-1)}}{\beta_{k}^{(i-1)}}, \\
\beta_{k}^{(i)}= & \sum_{\bar{k} \in \mathcal{K}_{g_{k}}^{(i-1)} \mid\{k\}}\left|\overline{\mathbf{h}}_{b_{k}, g_{k}, k}^{\mathrm{H}} \mathbf{w}_{\bar{k}}^{(i)}\right|^{2}+\sum_{\bar{g} \in \mathcal{G}_{b} \backslash\left\{g_{k}\right\}} \sum_{\bar{k} \in \mathcal{K}_{\bar{g}}}\left|\overline{\mathbf{h}}_{b_{k}, \bar{g}, k}^{\mathrm{H}} \mathbf{w}_{\bar{k}}^{(i)}\right|^{2}+\sum_{\bar{b} \in \mathcal{B} \backslash\left\{b_{k}\right\}} \sum_{\bar{g} \in \mathcal{G}_{\bar{b}}} \sum_{\bar{k} \in \mathcal{K}_{\bar{g}}}\left|\overline{\mathbf{h}}_{\bar{b}, \bar{g}, k}^{\mathrm{H}} \mathbf{w}_{\bar{k}}^{(i)}\right|^{2}+N_{0}, \\
\gamma_{k}^{(i)}= & \frac{\left|\overline{\mathbf{h}}_{b_{k}, g_{k}, k}^{\mathrm{H}} \mathbf{w}_{k}^{(i)}\right|^{2}}{\beta_{k}^{(i)}}
\end{aligned}
$$


plexity associated with the computation of the IBF vectors $\left\{\mathbf{w}_{k}\right\}_{k \in \mathcal{K}}$ at each iteration is $\mathcal{O}\left(K \times \Delta \times\left(S_{g}^{3}+S_{g}^{2}\right)\right)$, where $\Delta$ is the total number of bisection searches required to satisfy the per-group transmit power constraint [10]. As an example, for $N_{\mathrm{T}}=64, S_{g}=16$, and $G^{(b)}=4$, the group-specific IBF design yields a complexity reduction of $93.5 \%$ with respect to the ideal antenna-specific design without sacrificing much on the performance (as shown in Section IV).

\section{B. Distributed IBF Design with Limited Backhaul Signaling}

The main drawback of the centralized IBF design described in Section III-A is that it requires full CSI knowledge of the whole network. In this section, we propose a distributed IBF design to reduce the burdensome signaling associated with the CSI acquisition. Note that, to carry out the iterative optimization in (9) in a distributed fashion, the BSs need to periodically exchange information via backhaul signaling at each iteration in order to tackle the ICI, whereas the IGI terms can be handled locally. Under stringent backhaul constraints, the information exchange between the BSs can be further limited by allowing the ICI updates to occur on a longer-term basis with respect to the local IGI updates.

Considering the iterative solution of the centralized IBF design in (9), we observe that the following information is not available locally at $\mathrm{BS} b \in \mathcal{B}$ :

1) the dual variables $\left\{q_{\bar{k}}\right\}_{\bar{k} \in \mathcal{K} \backslash \mathcal{K}^{(b)}}$, which are necessary to update the IBF vector $\mathbf{w}_{k}^{(i)}$ in (9b);

2) the ICI caused by the neighboring BSs $\bar{b} \in \mathcal{B} \backslash\{b\}$, which is necessary to update the interference-plus-noise term $\beta_{k}^{(i)}$ in (9c).

For the latter, we rewrite $(9 \mathrm{c})$ as

$$
\begin{array}{r}
\beta_{k}^{(i)}=\sum_{\bar{k} \in \mathcal{K}_{g_{k}} \backslash\{k\}}\left|\overline{\mathbf{h}}_{b_{k}, g_{k}, k}^{\mathrm{H}} \mathbf{w}_{\bar{k}}^{(i)}\right|^{2}+\sum_{\bar{g} \in \mathcal{G}_{b} \backslash\left\{g_{k}\right\}} \sum_{\bar{k} \in \mathcal{K}_{\bar{g}}}\left|\overline{\mathbf{h}}_{b_{k}, \bar{g}, k}^{\mathrm{H}} \mathbf{w}_{\bar{k}}^{(i)}\right|^{2} \\
+\sum_{\bar{b} \in \mathcal{B} \backslash\left\{b_{k}\right\}} \zeta_{\bar{b}, k}^{(i)}+N_{0}
\end{array}
$$

where we have introduced

$$
\zeta_{\bar{b}, k}^{(i)}=\sum_{\bar{g} \in \mathcal{G}_{\bar{b}}} \sum_{\bar{k} \in \mathcal{K}_{\bar{g}}}\left|\overline{\mathbf{h}}_{\bar{b}, \bar{g}, k}^{\mathrm{H}} \mathbf{w}_{\bar{k}}^{(i)}\right|^{2}
$$

as the ICI caused by BS $\bar{b} \in \mathcal{B} \backslash\{b\}$ to UE $k \in \mathcal{K}^{(b)}$. Hence, to enable a distributed IBF design, each BS $b \in \mathcal{B}$ needs to receive $\left\{q_{\bar{k}}^{(i)}\right\}_{\bar{k} \in \mathcal{K}^{(\bar{b})}}$ and $\left\{\zeta_{\bar{b}, k}^{(i)}\right\}_{k \in \mathcal{K}^{(b)}}$ from the neighboring BS $\bar{b} \in \mathcal{B} \backslash\{b\}$ by means of backhaul signaling, which amounts to $K-\left|\mathcal{K}^{(b)}\right|$ (for the dual variables) plus $(B-1)\left|\mathcal{K}^{(b)}\right|$ (for the ICI terms) real-valued scalars at each iteration $i$. To reduce the amount of information exchange among the BSs, one can allow the backhaul signaling to occur every $M \geq 1$ iterations. This is formalized in Algorithm 1, where we assume that convergence is reached when $\left|\gamma_{k}^{(i)}-\gamma_{k}^{(i-1)}\right| \leq \epsilon, \forall k \in \mathcal{K}$. The convergence proof is similar to that provided in [13] and is thus omitted. Lastly, the computational complexity of such distributed IBF design is the same as its centralized counterpart (discussed at the end of Section III-A), since it is dominated by the computation of the IBF vectors.

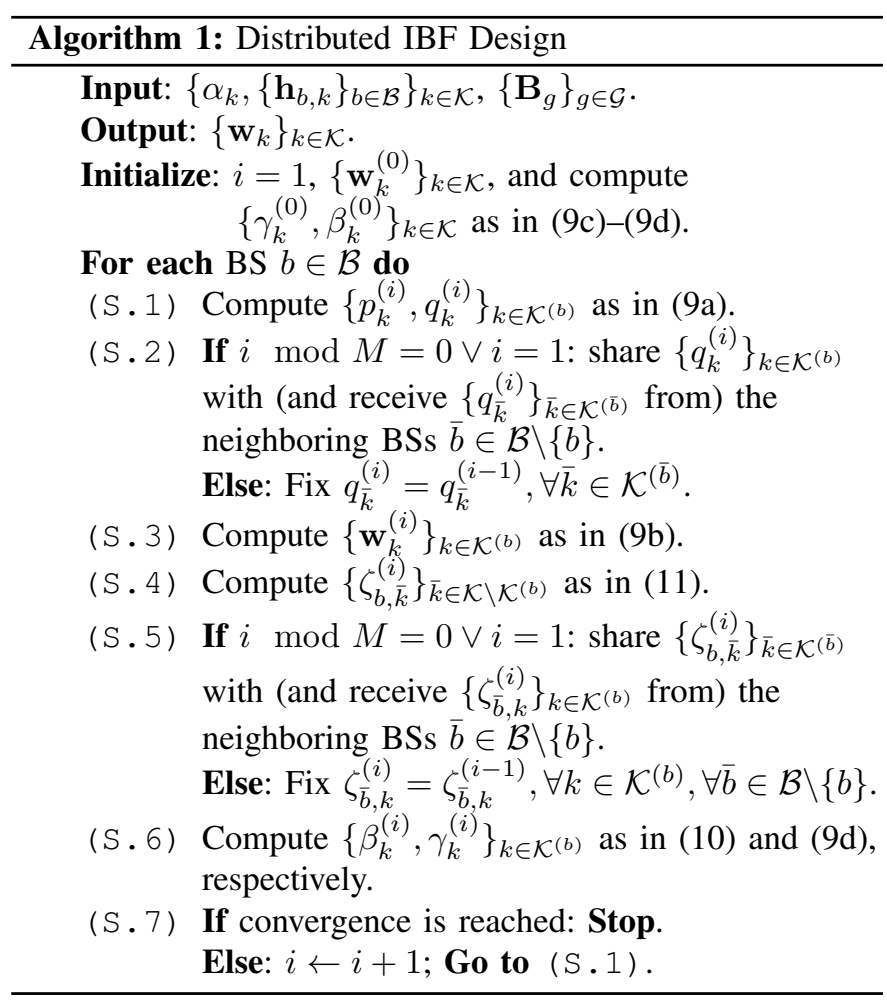

\section{NuMERICAL RESUlTS}

We consider a multi-cell massive MIMO system with $B=3$ BSs, each equipped with $N_{\mathrm{T}}=64$ antennas, serving a total of $K=48$ single-antenna UEs. The BSs are located as shown in Fig. 1 with inter-site distance of $100 \mathrm{~m}$. Each BS $b \in \mathcal{B}$ serves $\left|\mathcal{K}^{(b)}\right|=16$ UEs clustered into $G^{(b)}=4$ non-overlapping groups, where each group $g \in \mathcal{G}_{b}$ includes $\left|\mathcal{K}_{g}\right|=4$ UEs spanning an angle of $\pi / 6$. According to the channel model described in Section II, we generate the channels of each UE $k \in \mathcal{K}$ assuming $L=20$ scatterers and angular spread $\sigma_{b_{k}, k}=\pi / 12$. To mimic a cell-edge setting, all the UEs are placed at a distance of $40 \mathrm{~m}$ from the serving BS, and we further assume non-line-of-sight propagation with pathloss exponent $\eta=4$. Each BS $b \in \mathcal{B}$ uses the same number of statistical beams, which are equally divided among its UE groups as $S_{g}=S^{(b)} / G^{(b)}, \forall g \in \mathcal{G}_{b}$. All the UEs are assigned the same scheduling priority by fixing $\alpha_{k}=1, \forall k \in \mathcal{K}$. Lastly, the transmit power at the BSs and the noise power at the UEs are fixed to $P_{\mathrm{T}}=0 \mathrm{dBW}$ and $N_{0}=-174 \mathrm{dBW}$, respectively. In the considered scenario, the proposed iterative IBF design reaches convergence after approximately 30 iterations. The following numerical results are obtained by averaging over 100 channel realizations.

Fig. 2 compares the average sum-rate performance of the group-specific centralized IBF design in (7) with its distributed counterpart described in Algorithm 1 as a function of the number of iterations between backhaul updates $M$ and with different values of $S^{(b)}$ (note that $M=1$ indicates backhaul updates at every iteration). As expected, we observe that the average sum rate increases with the number of statistical 


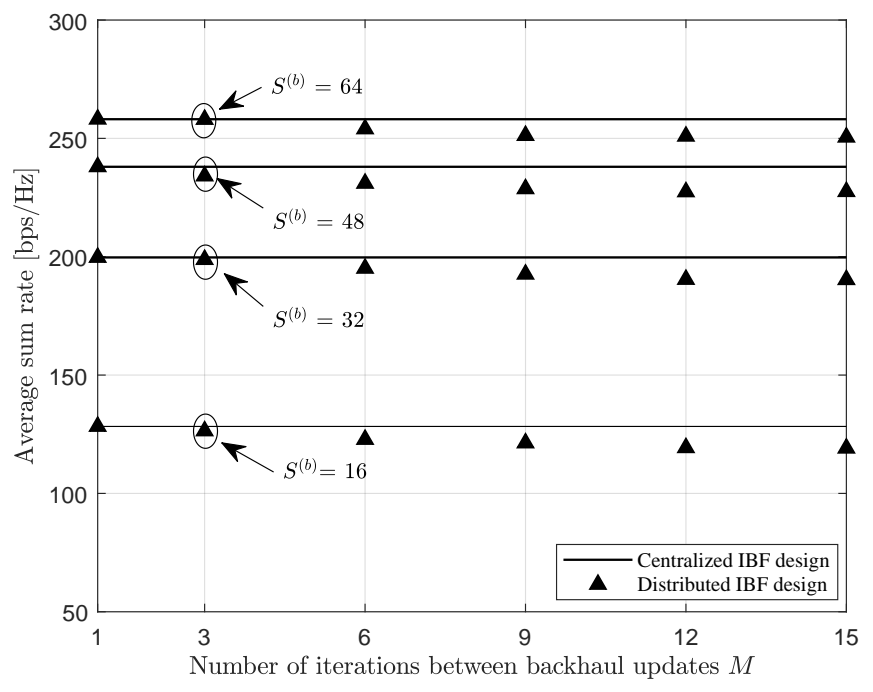

Fig. 2. Average sum rate against the number of iterations between backhaul updates.

beams and that the gap between centralized and distributed IBF design diminishes as $M$ decreases. In particular, allowing backhaul signaling every $M=9$ iterations yields a remarkably small performance loss compared to the case with $M=1$.

In Fig. 3, the centralized and distributed IBF designs (the latter with $M=9$ ) are compared with the fully-connected design described in [8]-[10], which is obtained by solving the problem in (7) without any UE grouping, and the corresponding curves are plotted against the number of statistical beams $S^{(b)}$. Again, the performance of Algorithm 1 with backhaul signaling every $M=9$ iterations performs remarkably well, especially for lower values of $S^{(b)}$. Furthermore, carrying out the optimization as if we were in a single-cell setting, i.e., by ignoring the ICI terms from the IBF expression in (9b), the average sum rate reduces significantly due to interference leakage. Lastly, if we ignore both the ICI and the IGI terms, the performance is further degraded only by a marginal extent. In fact, the ICI plays a vital role in the considered celledge scenario and overwhelms the IGI. For this reason, the ICI terms should be exchanged with sufficient frequency to improve the performance of Algorithm 1 (see Fig. 2).

\section{CONCLuSion}

In this paper, we propose a distributed, iterative method for downlink precoding in multi-cell massive MIMO systems with UE grouping. Considering two-stage precoding, we obtain the OBFs via greedy selection of DFT beams and we design the IBF vectors based on the resulting lower-dimensional effective channels. To this end, we present a distributed IBF design obtained by iteratively solving the KKT conditions of the WSRM problem. The proposed algorithm relies on limited backhaul signaling to periodically exchange ICI terms and dual variables among neighboring BSs, whereas the IGI within each BS is handled locally. Assuming stringent backhaul constraints, the ICI updates among the BSs are allowed to occur on a longer-term basis with respect to the local IGI

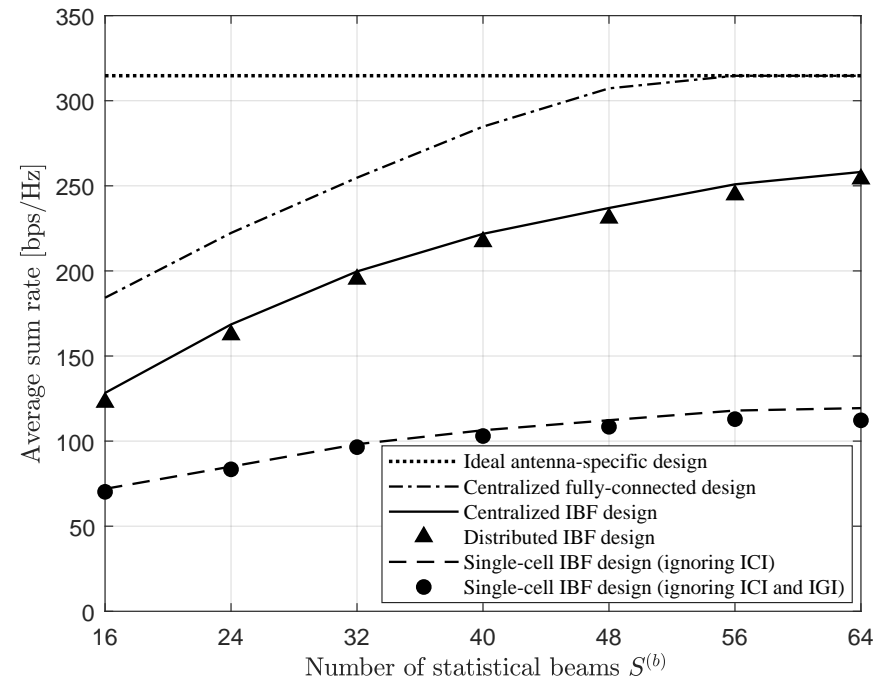

Fig. 3. Average sum rate against the number of statistical beams, with $M=9$ for the distributed IBF design.

updates. Our group-specific IBF design yields a remarkably small performance loss compared to the fully-connected design, while reducing the complexity by as much as $93.5 \%$ in the considered scenario.

\section{REFERENCES}

[1] T. L. Marzetta, "Non-cooperative cellular wireless with unlimited numbers of base station antennas," IEEE Trans. Wireless Commun., vol. 9, no. 11 , pp. 3590-3600, Nov. 2010.

[2] A. Adhikary, J. Nam, J.-Y. Ahn, and G. Caire, "Joint spatial division and multiplexing the large-scale array regime," IEEE Trans. Inf. Theory, vol. 59, no. 10 , pp. 6441-6463, Oct. 2013.

[3] A. F. Molisch, V. V. Ratnam, S. Han, Z. Li, S. L. H. Nguyen, L. Li, and K. Haneda, "Hybrid beamforming for massive MIMO: A survey," IEEE Commun. Mag., vol. 55, no. 9, pp. 134-141, Sept. 2017.

[4] J. Nam, A. Adhikary, J.-Y. Ahn, and G. Caire, "Joint spatial division and multiplexing: Opportunistic beamforming, user grouping and simplified downlink (DL) scheduling," IEEE J. Sel. Topics Signal Process., vol. 8, no. 5, pp. 876-890, Oct. 2014.

[5] J. Nam, Y.-J. Ko, and J. Ha, "User grouping of two-stage MU-MIMO precoding for clustered user geometry," IEEE Commun. Lett., vol. 19, no. 8, pp. 1458-1461, July 2015.

[6] Y. Xu, G. Yue, N. Prasad, S. Rangarajan, and S. Mao, "User grouping and scheduling for large scale MIMO systems with two-stage precoding," in Proc. IEEE Int. Conf. Commun. (ICC), Sydney, Australia, June 2014.

[7] A. Liu and V. Lau, "Hierarchical interference mitigation for massive MIMO cellular networks," IEEE Trans. Signal Process., vol. 62, no. 18, pp. 4786-4797, July 2014.

[8] A. Arvola, A. Tölli, and D. Gesbert, "Two-layer precoding for dimensionality reduction in massive MIMO," in Proc. IEEE European Signal Process. Conf. (EUSIPCO), Budapest, Hungary, Aug. 2016.

[9] A. Padmanabhan and A. Tölli, "An iterative approach for inter-group interference management in two-stage precoder design," in Proc. IEEE Global Commun. Conf. (GLOBECOM), Abu Dhabhi, UAE, Dec. 2018.

[10] —, "Interference management via user clustering in two-stage precoder design," in Proc. IEEE Int. Workshop Signal Process. Adv. in Wireless Commun. (SPAWC), Kalamata, Greece, June 2018.

[11] A. F. Molisch, Wireless Communications. John Wiley \& Sons, 2012.

[12] G. Scutari, F. Facchinei, and L. Lampariello, "Parallel and distributed methods for constrained nonconvex optimization-Part I: Theory," IEEE Trans. Signal Process., vol. 65, no. 8, pp. 1929-1944, Apr. 2017.

[13] G. Venkatraman, A. Tölli, M. Juntti, and L.-N. Tran, "Traffic aware resource allocation schemes for multi-cell MIMO-OFDM systems," IEEE Trans. Signal Process., vol. 64, no. 11, pp. 2730-2745, June 2016. 